\title{
Cefuroxime, levofloxacin, esomeprazole, and bismuth as first-line therapy for eradicating Helicobacter pylori in patients allergic to penicillin
}

\author{
Zhiqiang Song, Wei Fu and Liya Zhou*
}

\begin{abstract}
Background: Eradicating Helicobacter pylori infection is clinically challenging, notably in cases with penicillin allergy. Cephalosporin could be used in lieu of amoxicillin to eradicate Helicobacter pylori. The current work aimed to assess therapeutic efficacy and safety of a cefuroxime-based quadruple regimen in treatment-naïve individuals with penicillin allergy, as well as patient compliance.

Methods: In the present prospective single-center cohort study, 152 Helicobacter pylori infected individuals with penicillin allergy received eradication therapy with cefuroxime (500 mg twice/day), levofloxacin (500 mg once/day), esomeprazole (20 mg twice/day) and bismuth potassium citrate (220 mg twice/day; 14 days). Safety and compliance were evaluated 1 to 3 days upon eradication. The urea breath test was carried out 8 to 12 weeks upon eradication for efficacy assessment.

Results: This quadruple antimicrobial regimen eradicated the pathogen at 85.5\% (95\% confidence interval (Cl) 79.690.8\%), 88.4\% (95\% Cl 83.0-93.2\%) and 90.1\% (95\% Cl 85.2-94.4\%) in intention-to-treat, modified intention-to-treat and per-protocol analyses, respectively, with resistance rates of 4.6 and $40.0 \%$ in the background of cefuroxime and levofloxacin, respectively. Meanwhile, $21.3 \%$ of patients had adverse reactions, but none was serious. A total of $95.3 \%$ of patients showed good compliance. Poor compliance and cefuroxime resistance were detected by uni- or multivariate analyses as independent factors predicting therapeutic failure. Eradication rates in patients with dual levofloxacin and cefuroxime susceptibility, isolated levofloxacin resistance, isolated cefuroxime resistance and dual resistance were 97.2, 84.0, 50.0, and $0 \%$, respectively $(P=0.002)$.
\end{abstract}

Conclusions: Cefuroxime, levofloxacin, esomeprazole, and bismuth achieved decent efficacy, safety and compliance as first-line antimicrobial regimen in patients with Helicobacter pylori and penicillin allergy.

Keywords: Helicobacter pylori, Penicillin allergy, Cefuroxime, Levofloxacin, Eradication, Safety, Compliance

\section{Background}

Helicobacter pylori (H. pylori) infection and associated diseases are important global health problems. Eradication therapy is important in treating and preventing $H$. pylori infection-related diseases [1]. In recent years, $H$. pylori eradication has been increasingly difficult, mostly due to increased antibiotic resistance (e.g., to clarithromycin and metronidazole) and the limited availability of some antibiotics (e.g., tetracycline and furazolidone) in

\footnotetext{
* Correspondence: zhouliya123456@163.com

Department of Gastroenterology, Peking University Third Hospital, Beijing 100191, China
}

clinical practice [1-4]. Amoxicillin (semi-synthetic penicillin) is the core medicine used for $H$. pylori eradication due to its long-term low resistance rate [2-5]. However, approximately $5-10 \%$ of patients cannot take amoxicillin because of penicillin allergy, leading to more difficult $H$. pylori eradication in such cases [6-8]. According to the guidelines of Maastricht V Consensus Report, for patients allergic to penicillin, the proton pump inhibitor (PPI)-clarithromycin-metronidazole triple therapy is recommended as first-line eradication regimen in regions with low resistance to clarithromycin; in geographical areas with elevated resistance to clarithromycin, bismuth 
quadruple antimicrobial regimen is recommended [1]. Because of generally increased resistance to clarithromycin and metronidazole, PPI-clarithromycin-metronidazole therapy achieves unsatisfactory cure rates in most regions of the world [9-12]. In addition, due to a number of shortcomings (e.g., complicated administration, common adverse reactions, and tetracycline unavailability in many regions), the clinical application of bismuth quadruple therapy has been restricted [13-15]. Therefore, for penicillin-allergic patients, safe, effective and accessible regimens for $H$. pylori eradication are still lacking.

Cephalosporin and penicillin are $\beta$-lactam antibiotics, which share the same bactericidal mechanism (competitive inhibition of trans-peptidase, hindering peptidoglycan synthesis to suppress the biological functions or cause cell wall destruction in H. pylori) [16-18]. Antimicrobial sensitivity testing in vitro revealed a low resistance rate for cephalosporin in H. pylori (similar to that of amoxicillin) [19-21]. The limited data from a few clinical studies demonstrated that cephalosporin-containing regimens achieve relatively satisfactory eradication rates $[20,22,23]$. .These findings suggested that cephalosporin has a high potential for $\mathrm{H}$. pylori treatment. On the other hand, early observational studies have shown that approximately $10 \%$ of penicillin-allergic patients are also allergic to cephalosporin (cross-allergic phenomena) [24, 25]. However, with the accumulation of treatment experiences and further related researches, it is currently considered that the actual incidence of cross-allergy phenomena is very low $[6,8]$. Meanwhile, cross-allergy phenomena appear only in firstgeneration and some second-generation cephalosporins with the similar side-chain structure of penicillin; other cephalosporins could be safely applied in penicillin-allergic patients $[6,8,16,18]$. Moreover, cephalosporins are generally characterized by good safety and tolerance, as well as convenience and wide clinical application $[6,8,22,23]$. In view of these characteristics and advantages, it is reasonable to hypothesize that for penicillin-allergic patients, second or third generation cephalosporins without the penicillin-like side-chain structure could be potential effective alternatives to amoxicillin in eradicating $H$. pylori. To date, no related clinical study in this field has been reported.

Levofloxacin is usually used for rescue eradication of H. pylori infection $[1,3]$. However, recently published studies indicated that 14-day first-line treatment with amoxicillin-levofloxacin-PPI-bismuth still achieves satisfactory eradication rates in regions with high levofloxacin resistance $[20,26]$. This finding suggested that bismuth and levofloxacin have synergistic bactericidal effects. Bismuth increases the sensitivity of $H$. pylori to levofloxacin and, to a certain extent, facilitates the overcoming of resistance to the latter, which results in further improvement of the eradication efficacy [14, 26]. Moreover, our previous study showed that 14-day cefuroxime-levofloxacin-PPI-bismuth treatment results in a relatively good eradication rate $(89.8 \%$ in the per-protocol analysis) in patients without penicillin allergy [20]. Currently, clinical studies assessing levofloxacin-containing regimens for first-line $H$. pylori eradication in patients with penicillin allergy are unavailable.

Therefore, this work primarily aimed to assess the eradication efficacy of cefuroxime, levofloxacin, esomeprazole, and bismuth (CLEB) regimen in patients infected by $H$. pylori with penicillin allergy. We also evaluated safety and compliance for the above regimen, and analyzed risk factors that affect its efficacy in eradicating $H$. pylori.

\section{Methods}

\section{Patients and setting}

The current prospective cohort study was performed at the Gastroenterology clinic in a tertiary hospital of Beijing, China, from January 2015 to March 2017. Adult patients with dyspepsia and penicillin allergy were enrolled with clinically diagnosed $H$. pylori infection and no prior eradication treatment.

Exclusion criteria were: age $<18$ years; medicines with potential to affect results, including PPIs, $\mathrm{H}_{2}$-receptor blockers, bismuth salts, and antibiotics within 4 weeks; gastrointestinal cancer; a history of gastric or esophageal operation; severe concomitant disease; known allergy to any study drug; pregnancy or lactation in women.

\section{Ethical consideration}

Each patient provided signed informed consent. This trial had approval from the Ethics Committee of Peking University Third Hospital, Beijing, China, and conformed to the Declaration of Helsinki following Good Clinical Practice. All authors evaluated study results and approved the final manuscript.

\section{Study procedures}

Health care professionals at the Gastroenterology Unit provided a comprehensive explanation regarding the regimen and possible deleterious effects to the included patients. In addition, the patients were instructed orally and in writing regarding the importance of regular mediation intake, with recommendation to continue treatment even in case of mild or moderate adverse reactions and to call physicians for severe secondary effects. They were required to return within 3 days of eradication for the assessment of treatment compliance and adverse events. H. pylori eradication was evaluated 8-12 weeks upon treatment by the urea breath test (UBT; UCBT Kit, Atom High Tech, China). This was an open label trial.

Adverse events were determined by asking open-ended questions using patient self-reports and physical 
examinations, and grouped into the mild (no interference with daily routine), moderate (limited effects on daily routine), severe (marked effects on daily routine and medication discontinuation), and serious (death, hospitalization, disability, or required intervention for permanent damage prevention) types.

Compliance assessed by pill count was considered good ( $\geq 80 \%$ of pills taken) or poor $(<80 \%$ of drugs taken). Individuals poorly complying were not taken into account in the per-protocol (PP) analysis.

\section{Intervention}

The CLEB regimen consisted of cefuroxime $(500 \mathrm{mg}$ twice/day after breakfast and supper), levofloxacin (500 $\mathrm{mg}$ once/day after breakfast), esomeprazole (20 mg twice/day before breakfast and supper) and bismuth potassium citrate $(220 \mathrm{mg}$ twice/day before breakfast and supper) for 14 days.

\section{H. pylori detection}

Before enrolment, $H$. pylori infection was assessed as follows: (1) positive rapid urease test (RUT; HPUT-H102, San Qiang Bio \& Che, China) and histological WarthinStarry staining, and (2) positive UBT. Post-therapeutic H. pylori detection was by UBT 8 to 12 weeks upon eradication treatment. H. pylori infection was deemed eradicated with a single negative UBT.

In the patients who underwent upper endoscopy, gastric tissue biopsies obtained from the antrum were assessed by the RUT. In case of positive RUT, 2 biopsy samples from the antrum and corpus, respectively, were submitted to Warthin-Starry staining and H. pylori density evaluation. Two other samples from the antrum and corpus, respectively, were cultured for $H$. pylori and assessed for antibacterial sensitivity. Histological index grading was based on the updated Sydney system [27]. H. pylori density assessment was as + (sparse and sporadic), ++ (dense) and +++ (aggregated) distributions. The information about antibiotic resistance was employed for the analysis of parameters affecting eradication efficacy, but not for selecting first-line therapeutics. In case of CLEB therapy failure, the information was used to guide drug selection for second-line eradication therapy.

UBT was carried out following overnight fasting. Baseline breath samples were collected by blowing through plastic straws into $20-\mathrm{ml}$ containers, and capsules with $75 \mathrm{mg}$ of ${ }^{13} \mathrm{C}$-urea were provided to the patients with $100 \mathrm{ml}$ water. Then, breath samples were obtained 30 min later. A difference between baseline and 30-min samples exceeding 4.0 parts/1,000 of ${ }^{13} \mathrm{CO}_{2}$ as assessed on a gas isotope ratio mass spectrometer (GIRMS ZC202, Wan Yi Sci\& Tech, China) indicated positive results.

\section{H. pylori culture and antibacterial sensitivity test}

H. pylori was cultured or obtained from the gastric mucosa, and in vitro antibiotic resistance was evaluated by the Epsilometer test (AB Biodisk, Sweden) $[2,19]$. $H$. pylori strains with minimal inhibitory concentrations $(\mu \mathrm{g} / \mathrm{ml})$ of $>0.5,>0.5,>1,>8,>2$, and $>1$ were deemed to show resistance to amoxicillin, cefuroxime, clarithromycin, metronidazole, levofloxacin, and tetracycline, respectively [2, 5, 19, 27-30].

\section{Statistical analysis}

The sample size of 138 cases administered the CLEB regimen produced a $95 \%$ confidence interval $(\mathrm{CI})$ reflecting the sample proportion $\pm 5 \%$ with an estimated $H$. pylori first-line eradication rate of $90 \%$ (in a pilot trial conducted prior to the present investigation, successful eradication was achieved in 27/30 patients). Therefore, $\geq 152$ cased had to be enrolled to compensate for a $10 \%$ withdrawal rate.

The primary outcome was eradication rates in the intention-to-treat (ITT; all patients administered at least one drug dose), modified intention-to-treat (mITT; cases administered at least one drug dose and submitted to UBT), and PP (cases with complete adhesion to the trial protocol, except for poorly compliant individuals) analyses. Secondary outcomes included adverse event and compliance rates.

SPSS v18 (SPSS Inc., USA) was employed for all statistical analyses. $P<0.05$ indicated statistical significance. Categorical variables were presented as percentages or frequencies, and continuous ones as mean \pm SD. Eradication rates and 95\%CIs were determined. Pearson's chisquare or Fisher's exact test was employed to compare categorical variables. Univariate analysis was carried out to determine factors predicting $H$. pylori eradication after $\geq 1$ drug dose with the patient showing an endpoint. Multivariate logistic regression analysis (backward modeling and likelihood ratio) was performed for variables statistically significant in univariate analysis.

Penicillin allergy definition included any of the following criteria: (1) a history of allergic reactions, such as fever, rash, skin itching, and anaphylactic shock after penicillin received by oral administration, muscular injection, and intravenous injection; (2) positive skin test. A total of $20 \mathrm{~min}$ after intradermal injection of $0.1 \mathrm{ml}$ of the penicillin skin reagent, the penicillin skin test was considered to be positive with any of the following signs: local skin uplift with red halos, subcutaneous induration with a diameter of more than $1 \mathrm{~cm}$, pseudopodia and itching around the red halos, skin rash all over the body, and anaphylactic shock.

Cigarette smoking was reflected by $>1$ cigarette pack/ week consumed in the past 6 months. Alcohol drinking was reflected by $>50 \mathrm{~g}$ of alcohol/day consumed in the 
past 6 months. After upper endoscopy, patients showing duodenal and/or gastric ulcers were considered to have peptic ulcer disease, whereas those with no ulcers were regarded as non-ulcer dyspepsia cases. In addition, the cases with dyspepsia not examined by upper endoscopy were considered as having uninvestigated dyspepsia.

\section{Results}

The study flowchart is displayed in Fig. 1. One hundred fifty-two cases were included and administered eradication treatment. Ten cases were excluded from the PP analysis due to loss to follow-up $(n=2)$, intolerance to study drugs $(n=3)$, protocol violation $(n=1)$, and poor compliance $(n=4)$. The baseline properties of all enrolled patients are listed in Table 1 . H. pylori was detected in 77 patients by upper endoscopy using the RUT and Warthin-Starry staining (these cases underwent $H$. pylori culture, antibacterial sensitivity test, and $H$. pylori density assessment) and 75 patients by the UBT ( $H . p y l-$ ori culture and antimicrobial sensitivity test were successfully performed in 65 patients or $84.4 \%$ ).

\section{Eradication rates}

CLEB treatment yielded eradication rates of $85.5 \%$ (95\% CI 79.6-90.8\%; 130 of 152 patients), $88.4 \%$ (95\% CI 83.0-93.2\%; 130 of 147 patients) and 90.1\% (95\% CI
85.2-94.4\%; 128 of 142) in ITT, mITT and PP analyses, with resistance rates of 4.6 and $40.0 \%$ in the background of cefuroxime and levofloxacin, respectively.

\section{Adverse effects and compliance}

A total of $32(21.3 \%)$ patients showed adverse reactions, with 20, 9 and 3 mild, moderate and severe types, respectively. Nevertheless, serious adverse reactions were not observed. Adverse reactions are summarized in Table 2. Good compliance was achieved in 143 (95.3\%) cases.

\section{Parameters affecting eradication efficacy}

In univariable analysis, eradication rate was markedly elevated in cases with good compliance compared with the non-compliance group $(90.1 \%$ vs. $40.0 \%, P<0.001)$. Eradication rate was equally affected by cefuroxime resistance $(91.8 \%$ vs. $33.3 \%, P=0.002)$. Age, gender, body mass index, cigarette smoking, alcohol drinking, diagnoses, $H$. pylori density, and levofloxacin resistance did not significantly affect the eradication rate (Table 3 ). Multivariable analysis further revealed that poor compliance (odds ratio $(\mathrm{OR})=18.000,95 \% \mathrm{CI} 1.843-175.775, P=$ $0.013)$ and cefuroxime resistance (36.000, 2.500-518.371, $P=0.008)$ independently predicted therapy failure.

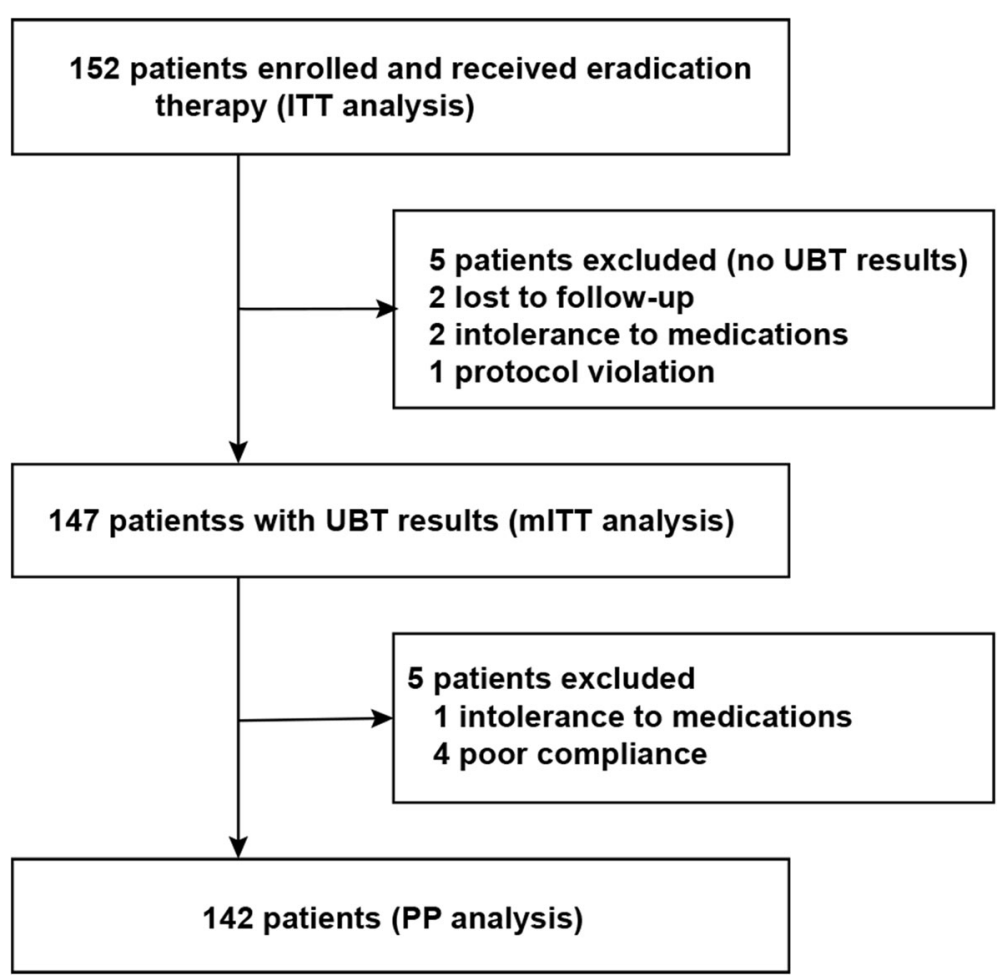

Fig. 1 Study flowchart ITT, intention-to-treat; mITT, modified intention-to-treat; PP, per-protocol; UBT, urea breath test 
Table 1 Patient baseline features

\begin{tabular}{ll}
\hline Variable & $\begin{array}{l}\text { Patients enrolled } \\
(n=152)\end{array}$ \\
\hline Age, mean \pm SD (years) & $42.8 \pm 13.7$ \\
Gender (female/male) & $70 / 82$ \\
Body mass index, mean \pm SD $\left(\mathrm{kg} / \mathrm{m}^{2}\right)$ & $23.1 \pm 2.8$ \\
Cigarette smoking (yes/no) & $24 / 128$ \\
Alcohol drinking (yes/no) & $28 / 124$ \\
Allergy to penicillin (skin $^{2}$ & $112 / 40$ \\
test positive/past allergy history) $_{\text {Diagnosis (PUD/NUD/UID) }}$ & $14 / 63 / 75$ \\
H. pylori density (+/++/+++) $^{\mathrm{a}}$ & $30 / 28 / 19$ \\
Ammoxicillin resistance (\%) $^{\mathrm{b}}$ & 3.1 \\
Cefuroxime resistance (\%) $^{\mathrm{b}}$ & 4.6 \\
Clarithromycin resistance (\%) $^{\mathrm{b}}$ & 38.5 \\
Metronidazole resistance (\%) $^{\mathrm{b}}$ & 63.1 \\
Levofloxacin resistance (\%) $^{\mathrm{b}}$ & 40.0 \\
Tetracycline resistance (\%) $^{\mathrm{b}}$ & 6.2
\end{tabular}

NUD Non-ulcer dyspepsia, PUD Peptic ulcer disease, UID Uninvestigated dyspepsia

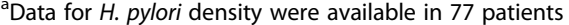

${ }^{\mathrm{b}}$ Data for $\mathrm{H}$. pylori antibacterial sensitivity were available in 65 patients

\section{Effects of antibiotic resistance on eradication success}

The impacts of cefuroxime and levofloxacin resistance on the success rate based on various probable resistance combinations are shown in Table 4. Eradication rates of cases showing dual cefuroxime and levofloxacin susceptibility, single levofloxacin resistance, isolated cefuroxime

Table 2 Adverse events and compliance

\begin{tabular}{ll}
\hline Variable, $n(\%)$ & $\begin{array}{l}\text { Eradication therapy } \\
(n=150)\end{array}$ \\
\hline Fatigue & $18(12.0)$ \\
Anorexia & $15(10.0)$ \\
Abdominal pain/discomfort & $14(9.3)$ \\
Nausea & $12(8.0)$ \\
Diarrhea & $12(8.0)$ \\
Headache & $5(3.3)$ \\
Vomiting & $3(2.0)$ \\
Skin rash & $2(1.3)$ \\
Taste distortion & $2(1.3)$ \\
Dizziness & $2(1.3)$ \\
Patients with adverse reactions & $32(21.3)$ \\
Mild adverse reactions & $20(13.3)$ \\
Moderate adverse reactions & $9(6.0)$ \\
Severe adverse reactions & $3(2.0)$ \\
(Medication discontinuation) & $143(95.3)$ \\
Good compliance &
\end{tabular}

Among the enrolled patients, two (one each for lost to follow-up and protocol violation) were not included in adverse effect and compliance assessments resistance, and dual cefuroxime and levofloxacin resistance were $97.2,84.0,50.0$, and $0 \%$, respectively $(P=0.002)$.

\section{Discussion}

H. pylori represents one of the major human pathogens. About $50 \%$ of all humans currently have $H$. pylori infection, with $5-10 \%$ of cases combined with penicillin allergy [1, 6-8]. Therefore, cases of $H$. pylori infection and penicillin allergy are relatively common and constitutes an important subgroup in $H$. pylori eradication strategies. Eradicating $H$. pylori in individuals allergic to penicillin is an important medical challenge. According to the recommendations of Maastricht $\mathrm{V}$ Consensus Report, PPI-clarithromycin-metronidazole and bismuth quadruple therapies are considered first-line eradication regimens in areas with low and high clarithromycin resistance, respectively [1]. Due to the small number of relevant studies and relatively low quality (Table 5) [9-12, 31-33], the degree of evidence is extremely low and the grade of recommendation is weak in the above guidelines. In addition, due to significantly increased resistance, PPI-clarithromycin-metronidazole therapy could not achieve satisfactory eradication efficacies in most regions of the world [9-12]. The clinical application of bismuth quadruple therapy has also been restricted due to complicated administration, frequent adverse reactions, and tetracycline unavailability in many regions [13-15]. Therefore, more related studies are required to establish a new safe, effective, and widely applied regimen for eradicating H. pylori.

In the present study, the CLEB regimen achieved satisfactory eradication efficacy $(85.5,88.4$, and $90.1 \%$ in ITT, mITT, and PP analyses, respectively) even in an area with high resistance (38.5\% to clarithromycin, $63.1 \%$ to metronidazole, and $40.0 \%$ to levofloxacin). Moreover, both safety (incidence of side effects of only about 20\%) and compliance (good compliance beyond 95\%) data were good. The majority of adverse events were transient and mild or moderate, and no overt cross-allergic reactions occurred. These findings provide a very promising new eradication regimen for $H$. pylori infected cases with penicillin allergy.

As mentioned above, cephalosporin could be a good alternative to amoxicillin for $H$. pylori eradication in patients with penicillin allergy. In this study, we used cefuroxime, mainly for the following reasons. (1) Cefuroxime and amoxicillin have an identical bactericidal mechanism through inhibition of cell wall synthesis [1618]. (2) Cefuroxime is active against $H$. pylori $[19,20]$. .The resistance rate in this study was only $4.6 \%$, similar to that of amoxicillin (3.1\%). (3) It is widely available in clinical practice. (4) Its safety and tolerance are exceedingly good. (5) Our previous study indicated that the CLEB regimen achieves relatively good eradication efficacy in patients 
Table 3 Variable analysis of factors affecting eradication efficacy

\begin{tabular}{|c|c|c|c|c|}
\hline \multirow[t]{2}{*}{ Variable, $n / \mathrm{N}(\%)$} & \multicolumn{2}{|l|}{ Univariable analysis } & \multicolumn{2}{|c|}{ Multivariable analysis } \\
\hline & Eradication efficacy $(n=147)$ & $P$ value & OR $(95 \% \mathrm{Cl})$ & $P$ value \\
\hline \multicolumn{5}{|l|}{ Age } \\
\hline$<35$ years & 40/45 (88.9) & 0.991 & & \\
\hline $35-55$ years & $59 / 67(88.1)$ & & & \\
\hline$>55$ years & $31 / 35(88.6)$ & & & \\
\hline \multicolumn{5}{|l|}{ Gender: } \\
\hline female & $61 / 68(89.7)$ & 0.655 & & \\
\hline male & $69 / 79(87.3)$ & & & \\
\hline \multicolumn{5}{|l|}{ Body mass index: } \\
\hline$<22.0 \mathrm{~kg} / \mathrm{m}^{2}$ & 38/42 (90.5) & 0.856 & & \\
\hline $22.0-25.0 \mathrm{~kg} / \mathrm{m}^{2}$ & 60/68 (88.2) & & & \\
\hline$>25.0 \mathrm{~kg} / \mathrm{m}^{2}$ & $32 / 37(86.5)$ & & & \\
\hline \multicolumn{5}{|l|}{ Cigarette smoking: } \\
\hline yes & 19/22 (86.4) & 0.742 & & \\
\hline no & 111/125 (88.8) & & & \\
\hline \multicolumn{5}{|l|}{ Alcohol drinking: } \\
\hline yes & $21 / 25(84.0)$ & 0.447 & & \\
\hline no & 109/122 (89.3) & & & \\
\hline \multicolumn{5}{|l|}{ Diagnosis: } \\
\hline PUD & $14 / 14(100.0)$ & 0.361 & & \\
\hline NUD & $53 / 61(86.9)$ & & & \\
\hline UID & 63/72 (87.5) & & & \\
\hline \multicolumn{5}{|l|}{ H. pylori density ${ }^{\text {a: }}$} \\
\hline+ & $25 / 29(86.2)$ & 0.929 & & \\
\hline $\begin{array}{l}++ \\
+++\end{array}$ & $\begin{array}{l}24 / 27(88.9) \\
17 / 19(89.4)\end{array}$ & & & \\
\hline \multicolumn{5}{|l|}{ Compliance: } \\
\hline good & 128/142 (90.1) & $<0.001$ & 18.000 & 0.013 \\
\hline poor & $2 / 5(40.0)$ & & $(1.843-175.775)$ & \\
\hline \multicolumn{5}{|c|}{ Cefuroxime resistance ${ }^{\mathrm{b}}$ : } \\
\hline susceptible & $56 / 61(91.8)$ & 0.002 & 36.000 & \\
\hline resistant & 1/3 (33.3) & & $(2.500-518.371)$ & 0.008 \\
\hline \multicolumn{5}{|c|}{ Levofloxacin resistance $^{\text {b: }}$} \\
\hline susceptible & $36 / 38(94.7)$ & 0.079 & & \\
\hline resistant & $21 / 26(80.8)$ & & & \\
\hline
\end{tabular}

CI Confidence interval, NUD Non-ulcer dyspepsia, OR Odds ratio, PUD Peptic ulcer disease, UID Uninvestigated dyspepsia

${ }^{a}$ Data for $H$. pylori density were available in 75 patients

${ }^{\mathrm{b}}$ Data for H. pylori antimicrobial sensitivity were available in 64 patients

Table 4 Effects of cefuroxime and levofloxacin resistance on Helicobacter pylori eradication

\begin{tabular}{lc}
\hline Variable, $n / \mathrm{N}(\%)$ & Eradication efficacy $(n=64)$ \\
\hline Cefuroxime susceptible and levofloxacin susceptible & $35 / 36(97.2)$ \\
Cefuroxime susceptible and levofloxacin resistant & $21 / 25(84.0)$ \\
Cefuroxime resistant and levofloxacin susceptible & $1 / 2(50.0)$ \\
Cefuroxime resistant and levofloxacin resistant & $0 / 1(0)$
\end{tabular}


Table 5 Clinical studies of first-line Helicobacter pylori eradication in patients allergic to penicillin

\begin{tabular}{|c|c|c|c|c|c|c|c|}
\hline Publication year & Country & Center & Number & Regimen & ITT cure rate & PP cure rate & Adverse reaction rate \\
\hline $2005[10]$ & Spain & single & 12 & $\mathrm{PPI}+\mathrm{CLA}+\mathrm{MET} 7$ days & $58 \%$ & $64 \%$ & $17 \%$ \\
\hline 2005 [31] & Puerto Rico & single & 17 & PPI + TET + MET 10 days & $84 \%$ & $84 \%$ & unavailable \\
\hline $2006[32]$ & Japan & single & 5 & PPI + TET + MET 7-14 days & $80 \%$ & $100 \%$ & unavailable \\
\hline 2010 [11] & Spain & multiple & 50 & $\mathrm{PPI}+\mathrm{CLA}+\mathrm{MET} 7$ days & $54 \%$ & $55 \%$ & $10 \%$ \\
\hline 2014 [33] & Japan & single & 11 & $\mathrm{PPI}+\mathrm{MET}+\mathrm{SIT} 7-14$ days & $100 \%$ & $100 \%$ & $64 \%$ \\
\hline \multirow[t]{2}{*}{$2015[9]$} & Spain & multiple & 112 & $\mathrm{PPI}+\mathrm{CLA}+\mathrm{MET} 7$ days & $57 \%$ & $59 \%$ & $14 \%$ \\
\hline & & & 50 & $\mathrm{PPI}+\mathrm{TET}+\mathrm{MET}+\mathrm{BIS} 10$ days & $74 \%$ & $75 \%$ & $14 \%$ \\
\hline \multirow[t]{4}{*}{2017 [12] } & Japan & single & 10 & $\mathrm{PPI}+\mathrm{CLA}+\mathrm{MET} 7$ days & $50 \%$ & $56 \%$ & unavailable \\
\hline & & & 13 & $\mathrm{VPZ}+\mathrm{CLA}+\mathrm{MET} 7$ days & $92 \%$ & $92 \%$ & unavailable \\
\hline & & & 20 & $\mathrm{PPI}+\mathrm{MET}+\mathrm{SIT} 7$ days & $100 \%$ & $100 \%$ & unavailable \\
\hline & & & 14 & VPZ + MET+SIT 7 days & $93 \%$ & $100 \%$ & unavailable \\
\hline
\end{tabular}

BIS Bismuth, CLA Clarithromycin, MET Metronidazole, ITT Intention-to-treat, PP Per-protocol, PPI Proton pump inhibitor, SIT Sitafloxacin, TET Tetracycline, VPZ Vonoprazan

without penicillin allergy, with good safety and compliance [20]; (6) As a second-generation cephalosporin, cefuroxime does not have a side chain structure similar to amoxicillin, showing no overt cross-allergy phenomenon $[6,8,16,18]$. The present study further confirmed that no obvious crossallergic effects were observed.

In the present study, another crucial antibiotic was levofloxacin, and the main reasons for its selection are described below. (1) Levofloxacin is broadly available in clinic. (2) Its overall safety and tolerability are good. (3) Recently published clinical studies have shown that 14-day amoxicillin-levofloxacin-PPI-bismuth quadruple antimicrobial treatment as a first-line regimen achieves satisfactory eradication rates even in areas showing elevated levofloxacin resistance [20, 26]. A study further by our group showed that 14-day cefuroxime-levofloxacin-PPI-bismuth quadruple antimicrobial treatment also yields relatively good eradication rates in patients without penicillin allergy [20]. Previous studies revealed that at levofloxacin resistance rates reaching 15-20\%, levofloxacin-containing triple regimens do not yield acceptable eradication efficacy $[5,26]$. In the current work, however, levofloxacin resistance rate reached $40 \%$, but the CLEB regimen still showed relatively good efficacy, which may be closely related to the inclusion of bismuth. On one hand, bismuth possesses antimicrobial properties itself $[14,34]$. On the other hand, bismuth helped overcome $H$. pylori resistance to levofloxacin, further improving eradication efficacy. This might be explained by that bismuth suppresses proton translocation to the bacterial cytoplasm and maintains intracellular $\mathrm{pH}$ at a level beneficial for metabolism and division in bacteria, enhancing the antibacterial efficacies of antimicrobials [34, 35].

As shown above, uni- and multi-variable analyses revealed antibiotic resistance and poor compliance as risk factors for failed eradication of $H$. pylori related disease, confirming that antibiotic sensitivity and treatment compliance represent the two top parameters determining successful $H$. pylori treatment $[1,36]$.

At present, tetracycline is difficult to obtain clinically in the mainland of China, so bismuth-containing quadruple therapy (bismuth, metronidazole, tetracycline and PPI) is rarely used. In the case of penicillin allergy, most patients were received two antibiotics from clarithromycin, levofloxacin and metronidazole for eradication. These three antibiotics have high resistance rates in the mainland of China, and the cure rate is poor and unsatisfactory. According to the relevant expert experiences and past study reports, the eradication rates were estimated only about $50-60 \%$ [9-12]. This study provides us a promising regimen for the patients allergic to penicillin. If the CLEB regimen fails, it will be very difficult. Tetracycline, furazolidone and rifabutin are difficult to obtain clinically, and the resistance rates of clarithromycin and metronidazole are very high. For the patients with penicillin allergy in the mainland of China, maybe other options could be chosen for the next eradication, such as minocycline/metronidazole containing quadruple regimen [37] or eradication therapy based on culture and susceptibility.

The present work had limitations: (1) All patients were from the same hospital, and multi-center trials in various world areas are required to confirm the present results. (2) The previous antibiotic use experience would affect the eradication rate, probably related to secondary antibiotic resistance from previous treatment $[1,17]$. Unfortunately, in this study we did not ask about the history of antibiotic use for other infectious diseases. In the future research, we can do an in-depth and accurate evaluation and discussion on this topic. (3) In addition, 
no control group was set. Therefore, it remains unknown whether any actual differences exist in eradication efficacy, safety, and compliance between this regimen and other recommended regimens for patents with penicillin allergy. Nevertheless, the present study provides an important reference for carrying out further related studies.

\section{Conclusions}

Overall, quadruple therapy with cefuroxime, levofloxacin, esomeprazole and bismuth achieves satisfactory eradication effectiveness, safety and compliance as firstline treatment in $H$. pylori infected cases with penicillin allergy.

\section{Abbreviations \\ H. pylori: Helicobacter pylori; PP: Per-protocol; PPI: Proton pump inhibitor}

\section{Acknowledgements}

None.

\section{Authors' contributions}

ZS contributed to study design, the clinical study, and manuscript preparation. WF contributed to the clinical study and experimental testing. LZ contributed to study design and manuscript editing. All authors have read and approved the final manuscript.

\section{Funding}

The current work was funded by the National Science \& Technology Pillar Program of the twelfth Five-Year Plan in China (2012BAI06B02), the National Natural Science Foundation of China (Grant No. 81670605), the Clinical key projects of Peking University Third Hospital (Y76493-03) and the Key laboratory for Helicobacter pylori infection and upper gastrointestinal diseases in Beijing (No. BZ0371). The funding body had no role in the design of the study and collection, analysis, and interpretation of data and in writing the manuscript.

\section{Availability of data and materials}

The datasets employed and/or analyzed in this study are available from the corresponding author upon reasonable request.

\section{Ethics approval and consent to participate}

Each patient provided signed informed consent. This trial had approval from the Ethics Committee of Peking University Third Hospital, Beijing, China, and conformed to the Declaration of Helsinki following Good Clinical Practice. All authors evaluated study results and approved the final manuscript.

\section{Consent for publication}

Not applicable.

\section{Competing interests}

The authors declare that they have no competing interests.

Received: 14 December 2018 Accepted: 21 July 2019

Published online: 25 July 2019

\section{References}

1. Malfertheiner P, Megraud F, O'Morain CA, Gisbert JP, Kuipers EJ, Axon AT, et al. Management of Helicobacter pylori infection-the Maastricht V/Florence consensus report. Gut. 2017;66(1):6-30.

2. Song Z, Zhang J, He L, Chen M, Hou X, Li Z, et al. Prospective multi-region study on primary antibiotic resistance of helicobacter pylori strains isolated from Chinese patients. Dig Liver Dis. 2014;46(12):1077-81.

3. Graham DY, Fischbach L. Helicobacter pylori treatment in the era of increasing antibiotic resistance. Gut. 2010:59(8):1143-53.

4. Megraud F, Coenen S, Versporten A, Kist M, Lopez-Brea M, Hirschl AM, et al. Helicobacter pylori resistance to antibiotics in Europe and its relationship to antibiotic consumption. Gut. 2013;62(1):34-42.
5. Graham DY. Hp-normogram (normo-graham) for assessing the outcome of H. pylori therapy: effect of resistance, duration, and CYP2C19 genotype. Helicobacter. 2016;21(2):85-90.

6. Macy E. Penicillin and beta-lactam allergy: epidemiology and diagnosis. Curr Allergy Asthma Rep. 2014;14(11):476.

7. Mirakian R, Leech SC, Krishna MT, Richter AG, Huber PA, Farooque S, et al. Management of allergy to penicillins and other beta-lactams. Clin Exp Allergy. 2015:45(2):300-27.

8. Pichichero ME, Zagursky R. Penicillin and cephalosporin allergy. Ann Allergy Asthma Immunol. 2014;112(5):404-12.

9. Gisbert JP, Barrio J, Modolell I, Molina-Infante J, Aisa AP, Castro-Fernandez $M$, et al. Helicobacter pylori first-line and rescue treatments in the presence of penicillin allergy. Dig Dis Sci. 2015;60(2):458-64.

10. Gisbert JP, Gisbert JL, Marcos S, Olivares D, Pajares JM. Helicobacter pylori first-line treatment and rescue options in patients allergic to penicillin. Aliment Pharmacol Ther. 2005;22(10):1041-6.

11. Gisbert JP, Perez-Aisa A, Castro-Fernandez M, Barrio J, Rodrigo L, Cosme A et al. Helicobacter pylori first-line treatment and rescue option containing levofloxacin in patients allergic to penicillin. Dig Liver Dis. 2010;42(4):287-90.

12. Ono S, Kato M, Nakagawa S, Mabe K, Sakamoto N. Vonoprazan improves the efficacy of Helicobacter pylori eradication therapy with a regimen consisting of clarithromycin and metronidazole in patients allergic to penicillin. Helicobacter. 2017;22(3):e12374.

13. Graham DY, Lee SY. How to effectively use bismuth quadruple therapy: the good, the bad, and the ugly. Gastroenterol Clin N Am. 2015;44(3):537-63.

14. Lu H, Zhang W, Graham DY. Bismuth-containing quadruple therapy for helicobacter pylori: lessons from China. Eur J Gastroenterol Hepatol. 2013; 25(10):1134-40.

15. Zhang W, Chen Q, Liang X, Liu W, Xiao S, Graham DY, et al. Bismuth, lansoprazole, amoxicillin and metronidazole or clarithromycin as first-line helicobacter pylori therapy. Gut. 2015;64(11):1715-20.

16. Campagna JD, Bond MC, Schabelman E, Hayes BD. The use of cephalosporins in penicillin-allergic patients: a literature review. J Emerg Med. 2012:42(5):612-20.

17. Furuta T, Graham DY. Pharmacologic aspects of eradication therapy for helicobacter pylori infection. Gastroenterol Clin N Am. 2010;39(3):465-80.

18. Pichichero ME, Casey JR. Safe use of selected cephalosporins in penicillin-allergic patients: a meta-analysis. Otolaryngol Head Neck Surg. 2007;136(3):340-7.

19. Bai $P$, Zhou LY, Xiao XM, Luo Y, Ding Y. Susceptibility of helicobacter pylori to antibiotics in Chinese patients. J Dig Dis. 2015;16(8):464-70.

20. Fu W, Song Z, Zhou L, Xue Y, Ding Y, Suo B, et al. Randomized clinical trial: esomeprazole, bismuth, levofloxacin, and amoxicillin or cefuroxime as firstline eradication regimens for helicobacter pylori infection. Dig Dis Sci. 2017; 62(6):1580-9.

21. Samra Z, Shmuely H, Niv Y, Dinari G, Passaro DJ, Geler A, et al. Resistance of helicobacter pylori isolated in Israel to metronidazole, clarithromycin, tetracycline, amoxicillin and cefixime. J Antimicrob Chemother. 2002:49(6):1023-6.

22. Fagoonee S, Astegiano M, Smedile A, Pellicano R. Efficacy of cefixime-based triple therapy for helicobacter pylori eradication: a retrospective study. Panminerva Med. 2013;55(3):309-10.

23. Tatsuta M, Ishikawa $H$, lishi H, Okuda S, Yokota $Y$. Reduction of gastric ulcer recurrence after suppression of helicobacter pylori by cefixime. Gut. 1990; 31(9):973-6.

24. Miranda A, Blanca M, Vega JM, Moreno F, Carmona MJ, Garcia JJ, et al. Cross-reactivity between a penicillin and a cephalosporin with the same side chain. J Allergy Clin Immunol. 1996;98(3):671-7.

25. Pichichero ME. Use of selected cephalosporins in penicillin-allergic patients: a paradigm shift. Diagn Microbiol Infect Dis. 2007;57(3 Suppl):13s-8s.

26. Liao J, Zheng Q, Liang X, Zhang W, Sun Q, Liu W, et al. Effect of fluoroquinolone resistance on 14-day levofloxacin triple and triple plus bismuth quadruple therapy. Helicobacter. 2013;18(5):373-7.

27. Liou JM, Chen CC, Chen MJ, Chen CC, Chang CY, Fang YJ, et al. Sequential versus triple therapy for the first-line treatment of helicobacter pylori: a multicentre, open-label, randomised trial. Lancet. 2013;381(9862):205-13.

28. Molina-Infante J, Romano M, Fernandez-Bermejo M, Federico A, Gravina AG, Pozzati L, et al. Optimized nonbismuth quadruple therapies cure most patients with Helicobacter pylori infection in populations with high rates of antibiotic resistance. Gastroenterology, 2013:145(1):121-128.e121.

29. Su P, Li Y, Li H, Zhang J, Lin L, Wang Q, et al. Antibiotic resistance of helicobacter pylori isolated in the southeast coastal region of China. Helicobacter. 2013;18(4):274-9. 
30. Zhou L, Zhang J, Song Z, He L, Li Y, Qian J, et al. Tailored versus triple plus bismuth or concomitant therapy as initial helicobacter pylori treatment: a randomized trial. Helicobacter. 2016;21(2):91-9.

31. Rodriguez-Torres M, Salgado-Mercado R, Rios-Bedoya CF, Aponte-Rivera E, Marxuach-Cuetara AM, Rodriguez-Orengo JF, et al. High eradication rates of helicobacter pylori infection with first- and second-line combination of esomeprazole, tetracycline, and metronidazole in patients allergic to penicillin. Dig Dis Sci. 2005;50(4):634-9.

32. Matsushima M, Suzuki T, Kurumada T, Watanabe S, Watanabe K, Kobayashi $\mathrm{K}$, et al. Tetracycline, metronidazole and amoxicillin-metronidazole combinations in proton pump inhibitor-based triple therapies are equally effective as alternative therapies against helicobacter pylori infection. J Gastroenterol Hepatol. 2006;21(1 Pt 2):232-6.

33. Furuta T, Sugimoto M, Yamade M, Uotani T, Sahara S, Ichikawa H, et al. Eradication of $\mathrm{H}$. pylori infection in patients allergic to penicillin using triple therapy with a PPI, metronidazole and sitafloxacin. Intern Med. 2014;53(6):571-5.

34. Dore MP, Lu H, Graham DY. Role of bismuth in improving helicobacter pylori eradication with triple therapy. Gut. 2016;65(5):870-8.

35. Marcus EA, Sachs G, Scott DR. Colloidal bismuth subcitrate impedes proton entry into helicobacter pylori and increases the efficacy of growthdependent antibiotics. Aliment Pharmacol Ther. 2015;42(7):922-33.

36. Graham DY, Lee YC, Wu MS. Rational Helicobacter pylori therapy: evidencebased medicine rather than medicine-based evidence. Clin Gastroenterol Hepatol. 2014;12(2):177-186.e173 Discussion e112-173.

37. Song ZQ, Zhou LY. Esomeprazole minocycline metronidazole and bismuth as first-line and second-line regimens for helicobacter pylori eradication. J Dig Dis. 2016;17(4):260-7.

\section{Publisher's Note}

Springer Nature remains neutral with regard to jurisdictional claims in published maps and institutional affiliations.

Ready to submit your research? Choose BMC and benefit from:

- fast, convenient online submission

- thorough peer review by experienced researchers in your field

- rapid publication on acceptance

- support for research data, including large and complex data types

- gold Open Access which fosters wider collaboration and increased citations

- maximum visibility for your research: over $100 \mathrm{M}$ website views per year

At $\mathrm{BMC}$, research is always in progress.

Learn more biomedcentral.com/submissions 